\section{MAIN DATABASE DEVELOPMENT}

The present database has been fully documented, including data models that describe the relationship between types of data, and the data dictionary that defines the individual data items. User requirements for the new database have been specified, and functional and technical specifications are being prepared. An audit of common reasons why records fail to load onto the current database will inform the development of the matching and code resolution algorithms for the new database. These algorithms ensure not only that people are correctly linked, but also that new notifications are correctly identified as new cancers or as re-notifications of cancers already known to the database.

\section{PROCESSING THE BACKLOG}

As at July 2000, there was a backlog of approximately 120,000 paper notifications filed alphabetically that require processing. An audit of CCR processes was undertaken to estimate average times for processing mail, sorting, coding, data entry and editing to estimate the resources required in order to eliminate the backlog in 12 months. ${ }^{6}$ The audit results will also be used to evaluate the effect of the introduction of a workflow management system. Additional staff have been appointed and the following timetable has been set: the 1998 Cancer Registry report produced by August 2001, the 1999 report by December 2001 and the 2000 report by June 2002. Monitoring of the progress indicates that the Registry will deliver these reports on time or earlier.

\section{REPORTING}

Consultations were held to gain advice from members of the Epidemiology Special Interest Group, Directors of Health Service Development, the Central Cancer Registry, and staff of the Cancer Epidemiology Research Unit, on a set of standards for reports. An improved Statistical Analysis Software (SAS) reporting module is nearing completion, which has a userfriendly series of drop down menus that present CCR data geographically, demographically, clinically, and by time period.

Up-to-date releases of an appropriately restricted CCR database, from which data that may identify an individual has been removed, will be produced quarterly for use with the annual reporting module. The quarterly module will be made available to authorised staff of the Cancer Council's Cancer Research and Registers Division. It may also be possible to make it more widely available to public health personnel in the NSW health system, through the Health Outcomes Information and Statistical Toolkit (HOIST), which is a public health data warehouse developed and maintained by the Epidemiology and Surveillance Branch of the NSW Department of Health.

A Web-based version of the reporting module, providing access to a large number of pre-prepared tables, is also being developed for the use of the general public. Both tables and graphs of data in age-specific categories and in age-standardised and crude form will be available, as well as new measures, such as person years of life lost due to cancer.

Ultimately, the redevelopment of the NSW Central Cancer Registry will result in the provision of up-to-date data on cancer incidence, mortality, prevalence and survival in flexible formats.

\section{REFERENCES}

1. NSW Department of Health. Central Cancer Registry business case for replacement Cancer Registry Systems. November 1997. Unpublished report.

2. Tracey E. Notification to the Central Cancer Registry-Outline of business requirements. Presented to the Patient Administration System (PAS) Consortium Meeting. December 7th 1999. Unpublished report.

3. CCR Specific Data items www.health.nsw.gov.au/iasd/dm/ isc/instructions/2000-2001/isc-instructions-2000-2001.html.

4. Health Information Exchange Cancer Feed Scope, version 1.11, October 2000.

5. Implementation of Health Level 7 (HL7) version 2.3.1 Part 4 Pathology results for registries. AS 4700.4 October 2000.

6. Tracey E. Resource requirements for processing the backlog of CCR notifications. May 2000. Unpublished report. W

\title{
TOWARDS A CLINICAL CANCER INFORMATION SYSTEM
}

Hanna Noworytko and Helen Moore

Epidemiology and Surveillance Branch

NSW Department of Health

\section{Bruce Armstrong}

Manager, Cancer Research and Registries

NSW Cancer Council
The efficient delivery of better health care depends on the availability of information on the outcomes of health care. The NSW Clinical Cancer Registries Project (ClinCRs) aims to improve — at a state level—-the collection, collation and analysis of data on patterns of cancer treatment and the health outcomes of cancer patients. 
The NSW ClinCRs collection system involves the 10 referral hospitals in NSW reporting a minimum data set to the NSW Central Cancer Registry (CCR). Subject to the success of the initial implementation, the system will be expanded to include all major non-metropolitan and district hospitals, both public and private. The CCR will process and analyse the data, and then return a collated state-level minimum data set to the contributing hospitals in a format of agreed indicators, together with an update on the vital statistics of patients treated by particular oncology departments. This article describes the content of the minimum data set and its relevance to improving cancer care.

The ClinCRs project is being conducted by the NSW Department of Health, and is overseen by 20 members of the Standing Committee of Directors of Cancer Services and Departments. In addition, advice from a wider group of cancer clinicians is being regularly canvassed. The objectives and processes of the ClinCRs implementation are fully compatible with the recommendations of the NSW Health Council. ${ }^{1}$

\section{CANCER CARE OBJECTIVES}

In 1997, the Optimising Cancer Management Expert Advisory Group defined the following objectives for cancer services:

- reduce incidence of cancer

- increase survival of cancer patients

- improve quality of life of cancer patients

- improve satisfaction with services for cancer patients and their carers

- improve equity of desirable outcomes

- increase cost-effectiveness of cancer services. ${ }^{2}$

\section{TABLE 1}

\section{CORE INDICATORS FOR CLINICAL CANCER REGISTRIES IN NSW ${ }^{3}$}

1. stage adjusted survival by time since diagnosis (absolute, relative or cause-specific).

2. distribution of patients by cancer type, stage and staging scheme used.

3. status of disease by time since diagnosis.

4. distribution of patients by performance status score.

5. status of disease at the end of definitive treatment.

6. severity adjusted treatment toxicity by toxicity type.

7. distribution of patients by treatment intent.

8. proportion of cases treated according to a local protocol based on evidence-based guidelines.

9. proportion of patients enrolled in clinical trials.

10. distribution of patients by treatment types.
The scope of the ClinCRs project is wide-ranging and includes monitoring survival, aspects of quality of life, and equity of care. Information from the ClinCRs, supported by other data, will be used to estimate changes in incidence of cancer, satisfaction with cancer services, and cost-effectiveness of those services.

\section{CORE INDICATORS FORTHE CLINICAL CANCER REGISTRIES}

Long-term and intermediate outcomes, and a vast array of interventions leading to the achievement of those outcomes, can be monitored by a number of indicators. Cancer care clinicians ranked 60 potential indicators, from which 10 were chosen on the basis of their importance and their ability to be collected. These have become the core indicators for the ClinCRs (Table 1). ${ }^{3}$

\section{MINIMUM DATA SET FOR THE CLINICAL CANCER REGISTRIES}

The ClinCRs system focuses on these 10 core indicators. To present them, 65 data items are required (Table 2); and these items have become the ClinCRs minimum data set. A full list of this minimum data set is included in the NSW Clinical Cancer Registers Minimum Data Set Data Dictionary, ${ }^{4}$ along with other data items that are needed to present detailed information about specific cancers.

The minimum data set makes good use of existing data. Of the 65 data items, 29 are already routinely collected at the state level, ${ }^{5}$ and 22 are collected in most local collections. A further 11 data items will be collected at the state-wide level. ${ }^{6}$ Linkage with other already established collections in the future has been considered. ${ }^{7,8}$

The ClinCRs minimum data set supports the NSW Cancer Services Model, which is a service delivery model that promotes high-quality integrated and coordinated cancer care. ${ }^{9}$ Information from the ClinCRs will stimulate improvements in the quality of cancer care by providing an overview of cancer in NSW.

\section{HOW THE MINIMUM DATA SET WILL IMPROVE CANCER CARE}

\section{Monitoring the patterns of courses of treatment by stage and other prognostic indicators}

Clinicians will be able to monitor prevailing patterns of treatment. For example, it will be possible to assess what types of cancer are treated by which surgical procedure, radiotherapy, chemotherapy (including oral chemotherapy); which types are receiving multimodality treatment, and in which order. This overview of treatment patterns is not presently available in NSW. The ClinCRs will report on survival and more immediate outcomes: 


\section{TABLE 2}

MINIMUM DATA SET FOR NSW CLINICAL CANCER REGISTRIES

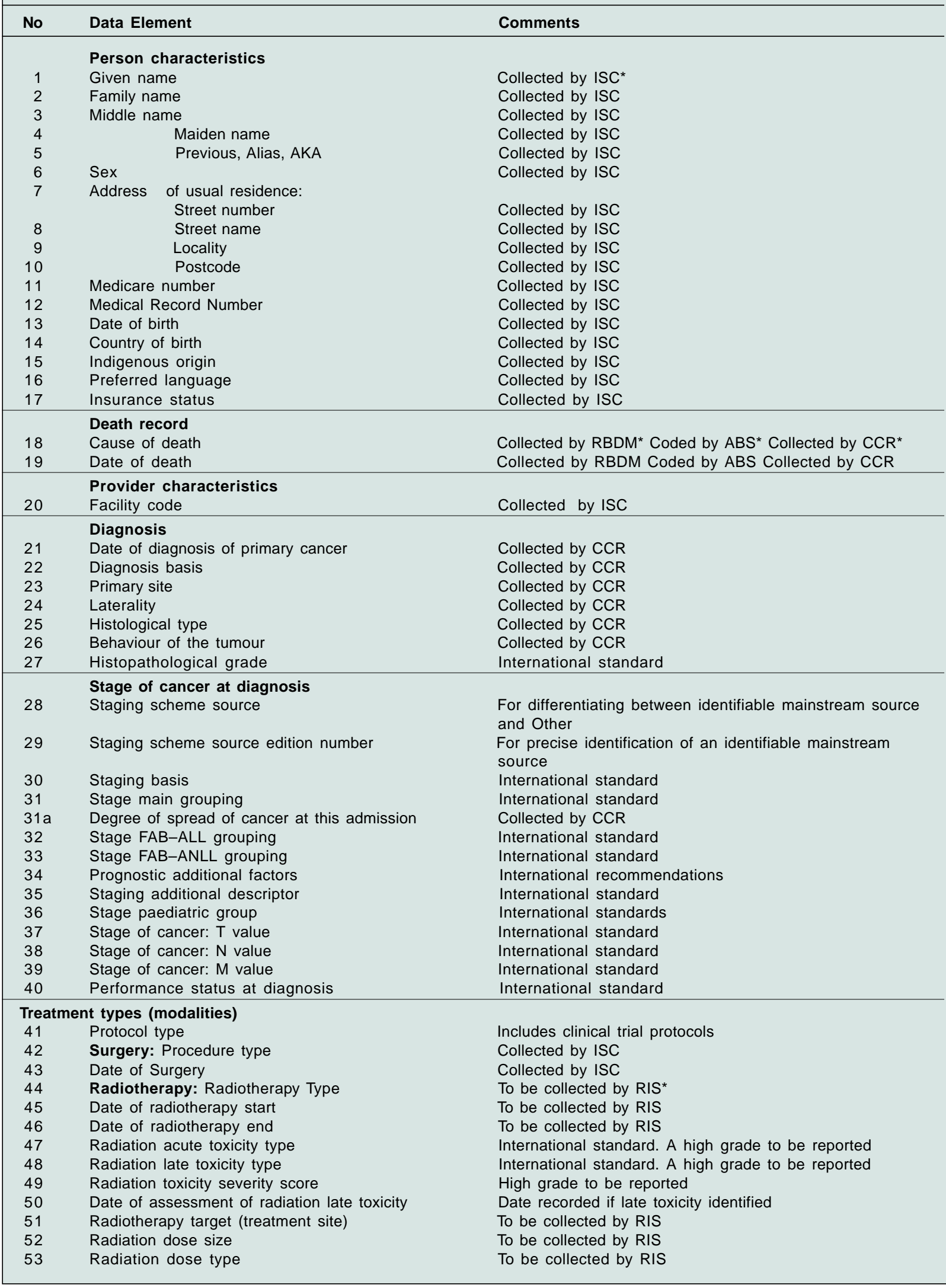


TABLE 2

MINIMUM DATA SET FOR NSW CLINICAL CANCER REGISTRIES (continued)

\begin{tabular}{|c|c|c|c|}
\hline No & Data Element & & Comments \\
\hline & Chemotherapy & & \\
\hline 54 & Date of chemotherapy start & & \\
\hline 55 & Date of chemotherapy end & & \\
\hline 56 & Chemotherapy toxicity type & & International standard. A high grade to be reported \\
\hline 57 & Chemotherapy toxicity severity score & & International standard. A high grade to be reported \\
\hline 58 & Date of assessment of chemotherapy late toxicity & & Date recorded if late toxicity identified \\
\hline 59 & $\begin{array}{l}\text { Chemotherapeutic agents standard treatment } \\
\text { protocol name (acronym) }\end{array}$ & & $\begin{array}{l}\text { Acronyms or a single agent name, as applicable. } \\
\text { Approach to be tested first in NSW and later replaced by } \\
\text { Anti-neoplastic agent name. }\end{array}$ \\
\hline $59 a$ & Anti-neoplastic agent name & & $\begin{array}{l}\text { Common coding reference: Antineoplastic drugs } \\
\text { (a manual maintained by SEER }{ }^{*} \text { in the United States). }\end{array}$ \\
\hline 60 & $\begin{array}{l}\text { Chemotherapeutic agent standard treatment } \\
\text { number of cycles }\end{array}$ & & $\begin{array}{l}\text { The number of times that a combination of agents } \\
\text { (protocol) was administered in the course of treatment }\end{array}$ \\
\hline \multicolumn{4}{|c|}{ Intermediate outcomes } \\
\hline 61 & Performance status at the end of treatment & & International standard \\
\hline 62 & Residual tumour & & International standard \\
\hline 63 & Date of diagnosis of recurrence & & \\
\hline 64 & Site of recurrence & & To be collected by RIS \\
\hline 65 & $\begin{array}{l}\text { Palliative care } \\
\text { Palliative care status }\end{array}$ & & Collected by ISC \\
\hline \multicolumn{4}{|c|}{ *Acronyms } \\
\hline ABS & Australian Bureau of Statistics & ISC & NSW Inpatient Statistics Collection \\
\hline ALL & Acute Lymphatic Leukaemia & RBDM & Registrar of Births, Deaths and Marriages \\
\hline ANLL & Acute Non-Lymphatic Leukaemia & RIS & Radiotherapy Information System \\
\hline CCR & NSW Central Cancer Registry & SEER & Surveillance, Epidemiology and End Results program \\
\hline FAB & French-American-British (staging system) & TNM & Tumour Nodes Metastasis, a cancer staging system \\
\hline
\end{tabular}

for example, duration of remission; the burden of serious side-effects by cancer type, stage, other prognostic indicators, and treatment pattern.

Clinical cancer registries with a wide population coverage are the best option for learning about patterns of treatment for rare cancers where data on treatment patterns and risk adjusted relative survival are very scarce.

\section{Comparing treatment results with other centres}

The ClinCRs overview of cancer care will be simple. Nevertheless, valid comparisons of results between regional centres and results at the state level can be made; and it will be possible to extend these comparisons to results achieved in other states and countries. Clinicians will evaluate ClinCRs reports and assess whether outcomes meet the expectations based on the scientific literature and other sources of information.

\section{Monitoring whether a recommended practice is being followed}

Clinicians will be able to assess whether patterns of treatment are consistent with the objective of high-quality cancer care and, in many instances, be able to compare their results with recommended evidence-based guidelines. For example, the treatment pattern for early breast cancer should reveal that, as recommended, the majority of cases (but not all cases) are treated with lumpectomy followed by a course of radiotherapy. Another example is monitoring the period of time between the date of diagnosis and surgery for melanoma, where rapid response is vital. Setting benchmarks may be considered in some instances.

\section{Improved ability to plan services and directing resources}

The ClinCRs information will allow an overview of the current cancer care workload on particular hospitals by cancer site, stage and related factors. Monitoring the trends in cancer care in different hospitals will be possible, as well as an assessment of the implications for resource allocation.

\section{The number of patients staged before the commencement of treatment should increase}

Staging, or determining as precisely as possible the extent of tumour spread both at the site of origin and as metastases, is a recognised prognostic tool in cancer management. ${ }^{10}$ Reviews of the medical records of cancer cases in the United States report significant improvements in the number of cases staged over the years. Overall increases in staging were as follows: 52.8 per cent 
of cases were staged in 1985 and 1986, 65 per cent in $1988,76.5$ per cent in $1990,84.2$ per cent in 1992,87 per cent in 1993 and 88 per cent in 1994..$^{10,11,12}$ Increased staging in hospitals was influenced by outside encouragement, and there was a continuous improvement in the quality, and percentage of cases staged. ${ }^{13}$

\section{The number of local treatment protocols may increase}

A treatment protocol for a condition describes the procedures, types, and doses of medication to be used for patient care. Protocols assist in the delivery of local treatment because of their accessibility, the condensed nature of their recommendations, and their portability between centres. Protocols may contain the recommendations of evidence-based guidelines, if such are available. Guidelines are considered to be 'slow' because they take a long time to develop, agree upon, ratify, and disseminate. They usually incorporate recommendations with reference to many elements in the manifestation of disease and the treatment continuum. New knowledge of evidence of effectiveness is often too fragmentary to be developed into ratified guidelines; however, it can be incorporated into local protocols, which are ideal vehicles for 'rapid response' to new information. The ClinCRs will be a convenient tool for monitoring evidence of effectiveness and adherence to local protocols.

\section{Recruitment in clinical trials may increase}

Poor recruitment to clinical trials in cancer care is a problem that is influenced by a variety of factors. The ClinCRs collection will show the levels of recruitment to trials in NSW, and may assist in developing strategies to assist with the recruitment of patients.

\section{Communication between different providers, and between providers and patients, will improve}

The ClinCRs collection offers definitions and classification systems which should assist with the development of a common cancer record for standardising and streamlining communication within cancer services, with primary care providers, and with patients.

\section{Support for the day-to-day management of cancer patients will be gained}

The NSW ClinCRs system will, with Central Cancer Registry assistance, identify patient outcomes that managing clinicians may otherwise find difficult to ascertain (such as death and long-term complications).

\section{CONCLUSION}

The ClinCRs minimum data set has a very broad application and the potential to assist in:
- providing the basis for monitoring the quality of services;

- service and resource planning;

- conducting clinical research;

- defining a common cancer record between specialities for day-to-day management of cancer patients.

\section{REFERENCES}

1. NSW Health Council. A better health system for NSW. Report of the NSW Health Council. Sydney: NSW Department of Health, 2000.

2. Epidemiology and Surveillance Branch. Optimising Cancer Management. Final Report to the Expert Advisory Group. Sydney: NSW Department of Health, April 1999.

3. Epidemiology and Surveillance Branch. Summary of the exercise to rank indicators for Clinical Cancer Registries in NSW. Sydney: NSW Department of Health, July 1999.

4. Epidemiology and Surveillance Branch. NSW Clinical Cancer Registries Minimum Data Set Data Dictionary. Edition 1: Draft for Comment. Sydney: NSW Department of Health, April 2000.

5. Information Management and Clinical Systems. Inpatient Statistics Collection Instruction Manual. Sydney: NSW Department of Health, 1999.

6. Radiotherapy Institutional Data Set Working Party and Radiotherapy Minimum Data Set Working Party. Draft Radiotherapy Minimum Data Set. Sydney: Statewide Services Development Branch, NSW Department of Health, 1998.

7. Australian Council on Healthcare Standards (ACHS) Care Evaluation Program. Clinical Indicators in Summary. Sydney: ACHS, 1998.

8. Centre for Health Service Development. The NSW AN-SNAP clinical training handbook. Sydney: NSW Department of Health, June 1999.

9. Cancer Care Model Working Party. A Cancer Care Model for NSW. Sydney: NSW Department of Health, 1999.

10. Fleming ID, Philips JL, Menck HR. The National Cancer Data Base report on completeness of American Joint Committee on Cancer staging in United States cancer facilities. Cancer 1996; 78: 1498-504.

11. Fleming ID, Philips JL, Menck HR, Murphy GP, Winchester DP. The National Cancer Data Base report on recent hospital cancer program progress towards complete American Joint Committee on Cancer-TNM staging. Cancer 1997; 80 (12): 2305-10.

12. Henson DE, Menck HR, Zuber-Ocweja KE. Trends in cancer patient staging. Steele GD, Winchester DP, Osteen RT, Menck HR, Murphy GP (editors). National Cancer Data Base: Annual review of patient care, 1994. Atlanta: American Cancer Society, 1994.

13. Smart CR, Eberle C, Simon S. An assessment of National Cancer Data Base quality control. Steele GD, Winchester DP, Menck HR, Murphy GP (editors). National Cancer Data Base: Annual review of patient care, 1993. Atlanta: American Cancer Society, 1993; 103-115. Fir 